\title{
Measurements of Permittivity, Dielectric Loss Tangent, and Resistivity of Float-Zone Silicon at Microwave Frequencies
}

\author{
Jerzy Krupka, Senior Member, IEEE, Jonathan Breeze, Anthony Centeno, Neil Alford, Thomas Claussen, and \\ Leif Jensen
}

\begin{abstract}
The complex permittivity and resistivity of float-zone high-resistivity silicon were measured at microwave frequencies for temperatures from 10 up to $400 \mathrm{~K}$ employing dielectric-resonator and composite dielectric-resonator techniques. At temperatures below $25 \mathrm{~K}$, where all free carriers are frozen out, loss-tangent values of the order of $2 \times 10^{-4}$ were measured, suggesting the existence of hopping conductivity or surface charge carrier conductivity in this temperature range. Use of a composite dielectricresonator technique enabled the measurement of materials having higher dielectric losses (or lower resistivities) with respect to the dielectric-resonator technique. The real part of permittivity of silicon proved to be frequency independent. Dielectric losses of high-resistivity silicon at microwave frequencies are mainly associated with conductivity and their behavior versus temperature can be satisfactory described by dc conductivity models, except at very low temperatures.
\end{abstract}

Index Terms-Conductivity measurement, dielectric losses, dielectric resonators, permittivity measurement, silicon, semiconductor materials measurements.

\section{INTRODUCTION}

$\mathbf{O}$ VER THE past 50 years, there have been numerous attempts to measure the complex permittivity of semiconductors at microwave frequencies employing waveguides, resonators, and broadband dispersive Fourier transform spectroscopic technique [1]-[7]. For the most accurate measurement of the complex permittivity or conductivity of semiconductors at microwave frequencies, it is essential that the sample under test has no electrical contact with any metal part of the fixture that is used for measurements. Some microwave techniques offer the possibility of contactless measurements, e.g., cylindrical resonant cavities and waveguides operating in one of the $\mathrm{TE}_{0}$ modes (usually the dominant one) [3]-[5]. For such structures, currents have only circumferential component and, thus, do not flow through the metal-semiconductor interface. Such a situation also occurs when the sample under test is separated

Manuscript received April 1, 2006; revised June 24, 2006.

J. Krupka is with the Instytut Mikroelektroniki i Optoelektroniki Politechniki Warszawskiej, 00-662 Warsaw, Poland (e-mail: krupka@imio.pw.edu.pl).

J. Breeze, A. Centeno, and N. Alford are with the Faculty of Engineering, Physical Electronics and Materials, London South Bank University, London SE1 0AA, U.K.

T. Claussen was with Topsil Semiconductor Material A/S, DK-3600 Frederikssund, Denmark. He is now with PBI-Dansensor, DK-4100 Ringsted, Denmark.

L. Jensen is with Topsil Semiconductor Material A/S, DK-3600 Frederikssund, Denmark.

Digital Object Identifier 10.1109/TMTT.2006.883655 from all metal parts of the fixture, e.g., [2]. In general, the complex permittivity of a semiconductor material is given by (1) as follows:

$$
\varepsilon=\varepsilon_{0}\left(\varepsilon_{r}-j \varepsilon_{r}^{\prime \prime}-j \frac{\sigma}{\omega \varepsilon_{0}}\right)=\varepsilon_{0} \varepsilon_{r}(1-j \tan \delta)
$$

where $\tan \delta$ is the effective dielectric loss tangent of the semiconductor given by (2) as follows:

$$
\tan \delta=\tan \delta_{d}+\frac{\sigma}{\omega \varepsilon_{0} \varepsilon_{r}}
$$

where $\varepsilon_{o}$ is the permittivity of the vacuum, $\varepsilon_{r}$ is the relative real permittivity of the semiconductor, $\omega$ is the angular frequency, $\sigma$ is conductivity, and $\tan \delta_{d}$ is the dielectric loss tangent associated with pure dielectric loss mechanisms (e.g., electronic and ionic polarization).

For doped semiconductors and for intrinsic semiconductors having energy gaps smaller than $1 \mathrm{eV}$, the dominant loss mechanism is related to the conductivity associated with free charge carriers up to high microwave frequencies and at temperatures that exceed the activation energy of dopands. For such materials, their dielectric loss tangent can be represented by the second term on the right-hand side of (2). High-resistivity float-zone silicon has found applications as a substrate material for various microwave devices such as transmission lines, filters, or antennas, especially at millimeter-wave frequencies [8]-[10]. Over the last years, significant progress has been achieved in growing silicon crystals with very large resistivities exceeding $10 \mathrm{k} \Omega \mathrm{cm}$; however, it is difficult to find in the literature or from manufacturers accurate measurement data of their complex permittivity, in particular as a function of temperature. This data is essential in the design of microwave devices. Such measurements are reported in this paper.

\section{Measurements TeChniQues}

Two measurement setups were used in this study and are shown in Fig. 1. In the first setup [see Fig. 1(a)], the sample under test was situated on a small single crystal quartz support inside a cylindrical cavity and the whole structure was mounted on the cold head of a closed cycle Gifford-McMahon cryocooler for low-temperature measurements or in an oven for elevated temperature measurements. Adjustable coupling mechanisms were used to control coupling coefficients from both ports of the resonator. The resonators were attached to the network analyzer via semirigid coaxial cables. In the second setup [see Fig. 1(b)], the same cylindrical cavity and single crystal 


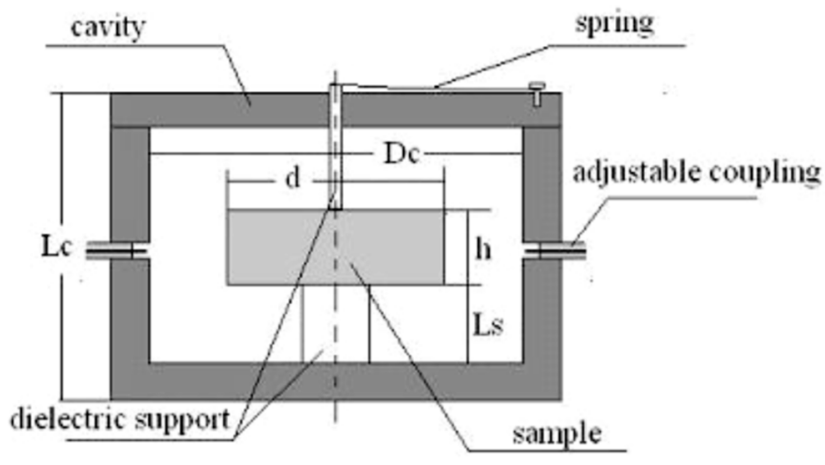

a)

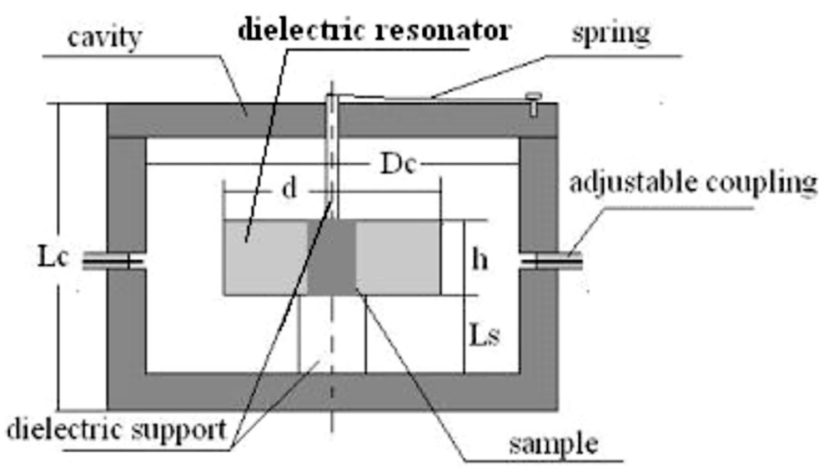

b)

Fig. 1. Sketch of measurement setups used in experiments. (a) Sample under test situated in cylindrical cavity. (b) Sample under test situated inside cylindrical dielectric resonator.

quartz support from the first configuration has been used, but the sample under test had a reduced diameter and was situated inside a ceramic dielectric ring resonator. The purpose of using a dielectric resonator configured in this way was to reduce the electric energy filling factor in the sample and, therefore, increase the $Q$ factor due to conductor losses in the silicon sample.

In the second measurement setup, the contribution to the $Q$ factor due to losses in the sample increased by one order of magnitude compared to the first measurement setup. This allows measurements of dielectric loss tangents one order of magnitude larger than those that can be measured in the first setup. High-resistivity silicon has dielectric loss tangents of the order of $10^{-2}-10^{-3}$ at room temperature and microwave frequencies. Therefore, techniques that have been used for measurements of its complex permittivity were the same as that used for dielectrics [11]-[14]. Most of the measurements were performed using the quasi- $\mathrm{TE}_{011}$ mode. This appears as the second resonance in the first measurement setup or the first resonant peak in the second. For some cryogenic measurements, other higher order modes have also been employed. In order to find the relationship between the measured resonant frequencies, $Q$ factors the real part of the relative permittivity, and dielectric loss tangent, it is necessary to rigorously solve Maxwell's equations for the structure under test. This was done using rigorous mode-matching and Rayleigh-Ritz techniques described in detail in [11]. In this paper, only the most important aspects of resonant techniques are explained. If the effective dielectric loss tangent of the sample is smaller than 0.1 , then the resonant frequencies depend on the real part of the permittivity and the dimensions of the resonant structure. In such a case, the real part of the permittivity can be evaluated from the measured resonant frequency of a specific mode taking into account thermal expansion of the resonant structure and the sample under test. The dielectric loss tangent of the sample can then be evaluated from

$$
\tan \delta=\left(Q_{u}^{-1}-Q_{p}^{-1}\right) / p_{e s}
$$

where $Q_{u}$ is the measured unloaded $Q$ factor of specific mode of resonator containing the sample under test, $Q_{p}$ is the $Q$ factor associated with parasitic losses in the cavity including metal wall losses and dielectric losses in the dielectric resonator and in the single crystal quartz support, and $p_{e s}$ is the electric energy filling factor for the sample under test (ratio of the electric energy stored in the sample to the electric energy stored in whole resonator)

$$
p_{e s}=\frac{\iiint_{V s} \varepsilon_{s}|\mathbf{E}|^{2} d v}{\iiint_{V t} \varepsilon(v)|\mathbf{E}|^{2} d v}
$$

where $V s$ is the volume of the sample, $V t$ is the volume of the whole resonant structure, $\varepsilon(v)$ is the spatially dependent permittivity inside the whole resonant structure, and $\varepsilon_{s}$ is the permittivity of the sample.

The $Q$ factor due to parasitic losses can be found from the following formulas:

$$
Q_{p}^{-1}=Q_{d}^{-1}+Q_{c}^{-1}
$$

where $Q_{d}^{-1}=p_{\mathrm{DR}} \tan \delta_{\mathrm{DR}}+p_{q s} \tan \delta_{q s}, p_{\mathrm{DR}}\left(p_{q s}\right)$ is the electric energy filling factors in dielectric resonator and in the single crystal quartz support, respectively, $\tan \delta_{\mathrm{DR}},\left(\tan \delta_{q s}\right)$ are the dielectric loss tangents of the dielectric resonator and the single crystal quartz support, respectively, and $Q_{c}$ is the $Q$ factor due to conductor losses in metal cavity walls

$$
Q_{c}^{-1}=R_{S} / G
$$

where $R_{S}$ is the surface resistance of metal cavity walls at a given frequency, $G$ is the geometric factor, which is defined as

$$
G=\omega \frac{\iiint_{V t} \mu_{0}|\mathbf{H}|^{2} d v}{\iint_{S}\left|\mathbf{H}_{\tau}\right| d s}
$$

$S$ is the internal surface of the cavity, and $\mathbf{H}_{\tau}$ is the component of the magnetic field tangential to the internal surface of the cavity.

The $Q$ factor associated with parasitic losses can be evaluated from measurements made of the unloaded $Q$ factors of the resonator without the silicon sample and of the empty cavity versus temperature. These measurements allow assessment of the surface resistance of the cavity walls and, in the second, experimental setup of the losses in the dielectric resonator, as a function of temperature. Losses in the single crystal quartz support are negligible due to the small electric energy filling factor value 
TABLE I

RESUltS OF ROOM-TEMPERATURE MEASUREMENTS. $D c=24.0 \mathrm{~mm}$, $L c=16.12 \mathrm{~mm}, d=15.455 \mathrm{~mm}(* 7.97 \mathrm{~mm}), h=6.0 \mathrm{~mm}, L s=4.26 \mathrm{~mm}$

\begin{tabular}{llllll}
\hline \hline Mode & $\mathrm{f}(\mathrm{GHz})$ & $\mathrm{Q}_{\mathrm{u}}$ & $\mathrm{Q}_{\mathrm{p}}$ & $\mathrm{p}_{\mathrm{e}}$ & $\tan \delta$ \\
\hline $\mathrm{TE}_{011}$ & 6.685 & 550 & 38950 & 0.9605 & 0.001866 \\
\hline $\mathrm{HE}_{111}$ & 6.797 & 1134 & 18030 & 0.4346 & 0.001901 \\
\hline$* \mathrm{TE}_{011}$ & 4.824 & 4036 & 16200 & 0.0885 & 0.002101 \\
\hline \hline
\end{tabular}

in it and the extremely low dielectric losses of quartz (below $2 \times 10^{-5}$ ). Once the material properties of metal cavity walls and dielectric parts in the cavity are known, the geometric factor and electric energy filling factors in the sample are numerically evaluated. It should be mentioned that, for high-resistivity silicon, the parasitic losses in the measurement setups are much smaller than losses in the silicon sample since effective dielectric loss tangent of silicon is usually larger than $10^{-3}$, except at very low temperatures. In this case, the effective dielectric loss tangent can be determined with approximately the same precision as a $Q$-factor measurement, i.e., approximately $1 \%-2 \%$. When dielectric loss tangent values in silicon samples are of the order of $10^{-5}$ or less (at very low temperatures), it is still possible to measure them very precisely by employing modes having large azimuthal mode indices, i.e., the so-called whispering-gallery modes. By employing whispering-gallery-mode techniques, dielectric loss tangents as low as $10^{-9}$ have been measured on high-purity single-crystal sapphire samples at cryogenic temperatures [12].

The upper limit for loss-tangent measurements is associated with the lowest $Q$-factor values that can be effectively measured, and the value of the electric energy filling factor. In our resonant cavity, the minimum value of the measurable $Q$ factor was approximately 100 . The electric energy filling factors for the two measurement setups are shown in Table I, together with room-temperature measurements results for the two samples.

As can be seen in Table I, the use of the quasi-HE $\mathrm{HE}_{111}$ mode enables the measurement of losses approximately twice as large as that obtainable using the quasi- $\mathrm{TE}_{011}$ mode. This is related to an electric energy filling factor value for the $\mathrm{HE}_{111}$ mode, which is twice as high as that of the $\mathrm{TE}_{011}$ mode. An electric energy filling factor in the sample can be further reduced by employing a dielectric ring resonator, as in the second measurement setup. In this case, the electric energy filling factor in the sample is reduced by an arbitrary number, which depends on the permittivity and external diameter of the dielectric resonator. The last row of Table I shows that, for the second measurement setup, the electric energy filling factor has been reduced by a factor of 10.

\section{RESULTS OF EXPERIMENTS}

\section{A. Measurements Employing Silicon Sample as the Dielectric Resonator}

Measurements at room temperature have been performed on two bulk cylindrical p-type silicon samples having diameters of $15.455 \mathrm{~mm}$ (Sample \#1) and $15.457 \mathrm{~mm}$ (Sample \#2) and a height of $6.0 \mathrm{~mm}$. The surfaces of the samples were mechanically polished, but not to optical quality. The real part of the

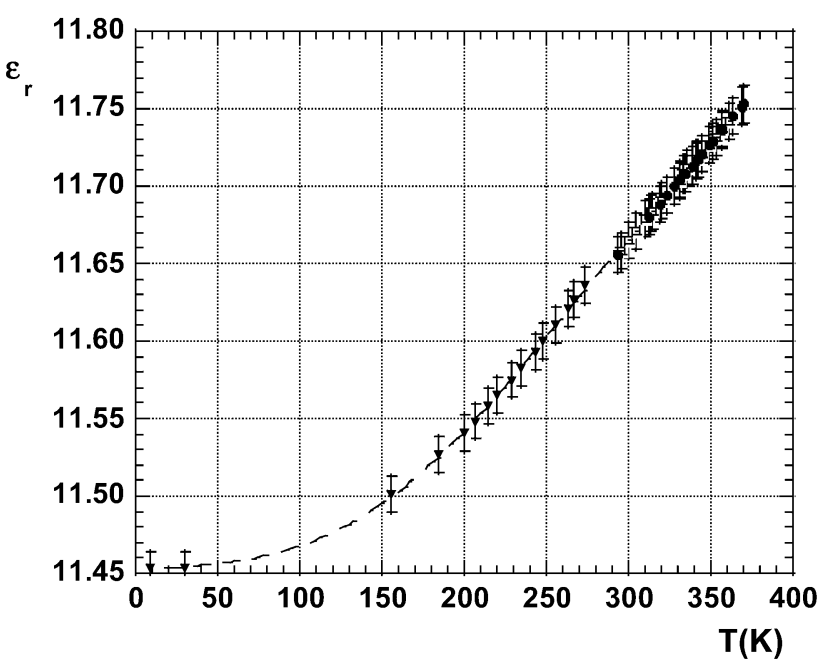

Fig. 2. Permittivity versus temperature for sample with $d=15.455 \mathrm{~mm}$. Experimental data points include results extracted from measurements employing quasi- $\mathrm{TE}_{011}$ mode in two cavities $(6.62$ and $6.69 \mathrm{GHz})$ and the third $\mathrm{TE}_{0}$ mode $(11.5 \mathrm{GHz})$ in the second cavity.

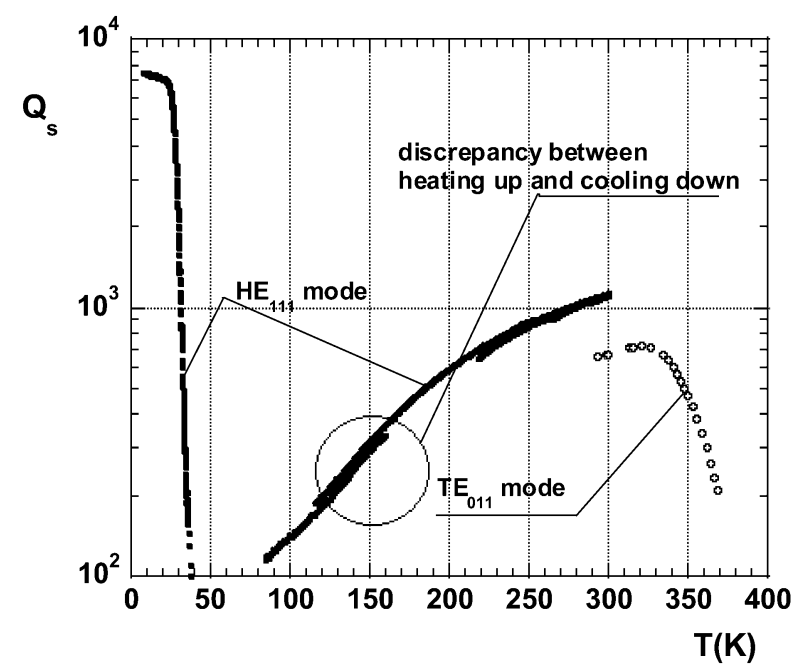

Fig. 3. $Q$ factors in the sample versus temperature for silicon sample \#1 with $d=15.455 \mathrm{~mm}$ measured at frequencies of approximately $6.8 \mathrm{GHz}\left(\mathrm{HE}_{111}\right.$ mode) and $6.62 \mathrm{GHz}\left(\mathrm{TE}_{011}\right.$ mode).

relative permittivity $\varepsilon_{r}$ was extracted for both samples at different frequencies by employing higher order $\mathrm{TE}_{0}$ modes. The results of these measurements showed the relative permittivity to be independent of frequency. In permittivity determination, the thermal expansion of the silicon samples and copper cavities were taken into account. Results of permittivity measurements as a function of temperature are shown in Fig. 2.

It is observed that results of experiments are very smooth with experimental errors in the range of $0.1 \%$. The experimental errors predominantly depend on dimensional uncertainties of the samples under test. Results of measurements of the $Q$ factor and dielectric loss tangent versus temperature for bulk silicon samples are presented in Figs. 3-6. In the temperature region of $100-250 \mathrm{~K}$, losses are associated with conductivity due to free holes. In this region, all Boron atoms are ionized, and the conductivity, and therefore, the dielectric loss tangent, depends on the mobility of the holes [16]. Measurements between 36-100 K 


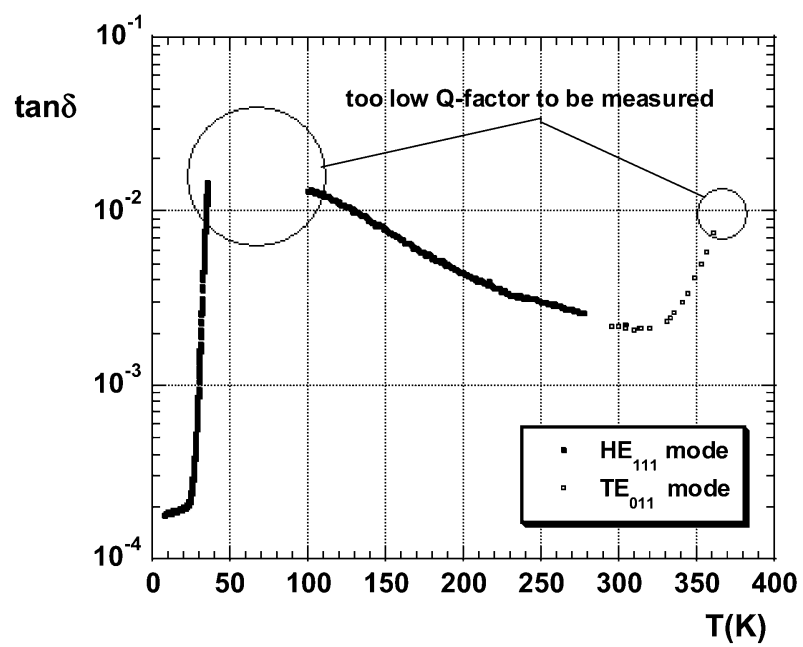

Fig. 4. Effective dielectric loss tangent versus temperature for silicon sample \#1 with $d=15.455 \mathrm{~mm}$ measured at frequencies of approximately $6.8 \mathrm{GHz}$ ( $\mathrm{HE}_{111}$ mode) and $6.62 \mathrm{GHz}\left(\mathrm{TE}_{011}\right.$ mode).

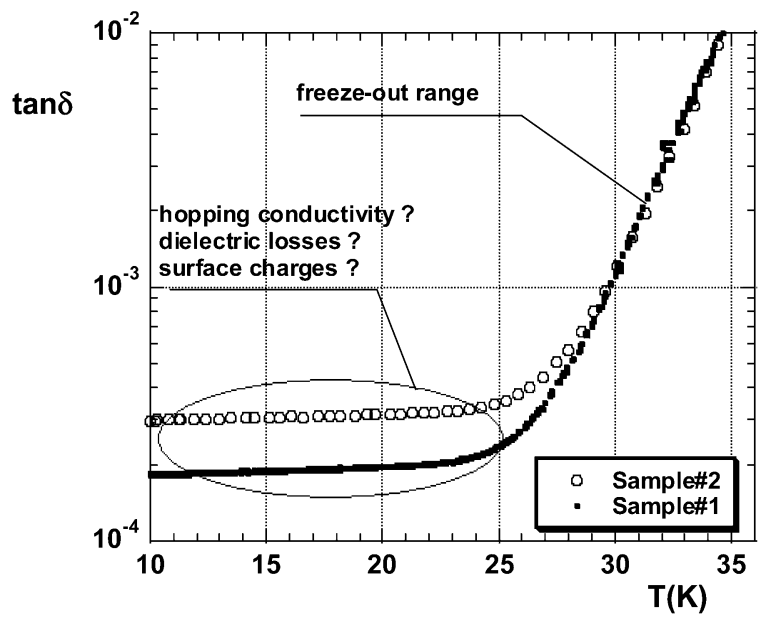

Fig. 5. Effective dielectric loss tangent versus temperature at low-temperature region for two silicon samples at frequency of approximately $6.8 \mathrm{GHz}\left(\mathrm{HE}_{111}\right.$ mode).

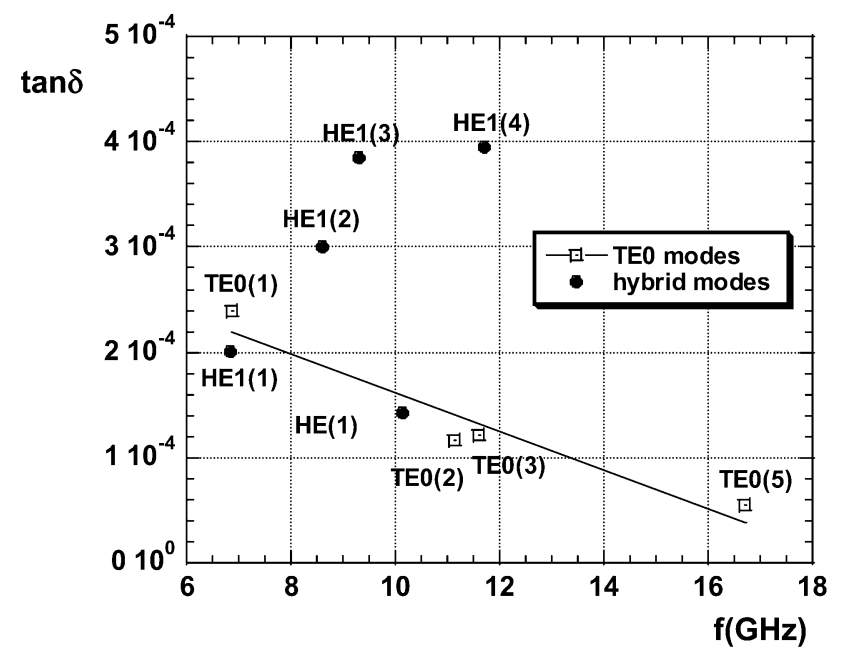

Fig. 6. Effective dielectric loss tangent of sample \#2 at $10 \mathrm{~K}$ evaluated using $Q$ factors of different modes.

on bulk silicon samples were not possible due to the $Q$-factor values being less than 100 .

Hysteresis effects are observed in the sample. This is manifested by measuring different $Q$ factors at the same temperature when the temperature is increasing compared to when it was decreasing. The increase in losses at temperatures above $310 \mathrm{~K}$ are related to the generation of free charge carriers through the energy gap, and above $350 \mathrm{~K}$, high-resistivity silicon becomes essentially intrinsic. In the temperature range of $25-40-\mathrm{K}$, hole freeze-out effects can be observed. It is not clear what the origin of dielectric losses are below $25 \mathrm{~K}$. To some extent, losses in this temperature range may be attributed to hopping conductivity [15], nonuniform dopand distribution in the sample, and accumulation of charge carriers on the surface of samples or dielectric losses. Additional measurements of losses at $10 \mathrm{~K}$ versus frequency have been performed employing several higher order modes. Results of these measurements are shown in Fig. 6 where the first number in the description of the modes denotes an azimuthal mode index, while the second one, in parenthesis, denotes the sequence of the mode on the frequency scale. In other words, both radial and axial mode indices are combined into one as they are not integer numbers for our resonant structure. In the literature, these indices are often denoted by Greek characters to underline this feature, or alternatively, such modes are termed "quasi." It is seen that dielectric loss tangent values measured with $\mathrm{TE}_{0}$ modes decrease with increasing frequency. This result supports the assumption about conductive loss mechanisms such as hopping conductivity. It should be mentioned that for $\mathrm{TE}_{0}$ modes, the electric field in the cavity (and sample) only has an azimuthal component that is tangential to all sample surfaces. This is not true for measurements based on the hybrid modes that have all three spatial components of the electric field, some of them perpendicular to the sample surfaces. The spatial distribution of the electric field for hybrid modes is complicated and unique for each mode. If conductivity is nonuniform or anisotropic in the sample volume, it may lead to different values of measured dielectric losses for each mode, as seen in Fig. 6.

It should also be noted that measurement errors employing $\mathrm{TE}_{0}$ modes at $10 \mathrm{~K}$ are small, around $2 \%$ or less, because all resonances have $Q$ factors of the order of a few thousand, and they are well separated in frequency and easily identified. Measurements performed in the same cavity on a single-crystal $\mathrm{MgO}$ sample at $10 \mathrm{~K}$ had shown dielectric loss tangent values below $1 \times 10^{-6}$.

\section{B. Measurements Employing Composite Dielectric-Resonator Technique}

For these measurements, the diameter of sample \#1 is reduced creating sample \#3. Sample \#3 has a diameter of $7.97 \mathrm{~mm}$ and a height of $6.00 \mathrm{~mm}$. A cylindrical ring dielectric resonator was manufactured from $\mathrm{Ba}\left(\mathrm{Zn}_{1 / 3} \mathrm{Ta}_{2 / 3}\right) \mathrm{O}_{3}$ (BZT) ceramic. It has the same height as that of the silicon sample, an external diameter of $16.14 \mathrm{~mm}$, and an internal diameter of $8.00 \mathrm{~mm}$. The permittivity of the ceramic dielectric is 29.86 and the dielectric loss tangent $\tan \delta$ is $5.9 \times 10^{-5}$ at $10 \mathrm{GHz}$.

For reference, measurements of parasitic losses versus temperature when a PTFE sample was inserted instead of the silicon sample were undertaken. The PTFE sample was used to keep the BZT resonator in a fixed position. This was necessary due to the vibrations of the close-cycle helium refrigerator. A room-temperature measurement showed that the $Q$ factor and resonant 


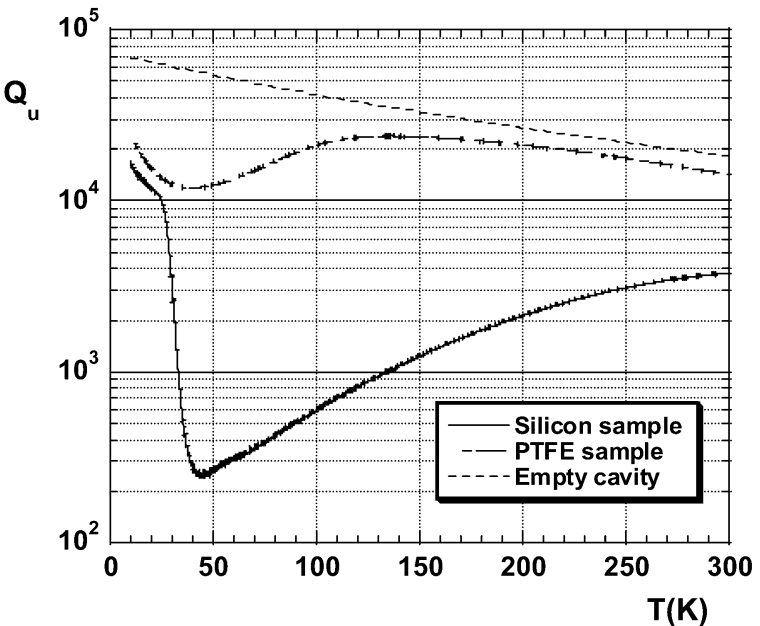

Fig. 7. Unloaded factors versus temperature for quasi-TE $E_{011}$ mode of BZT dielectric resonator containing silicon sample \#3 and PTFE sample having $d=$ $7.97 \mathrm{~mm}$ at a frequency of approximately $4.98 \mathrm{GHz}\left(\mathrm{TE}_{011}\right.$ mode). $Q$ factor of the empty cavity without BZT dielectric resonator. Support is additionally denoted via the dotted line.

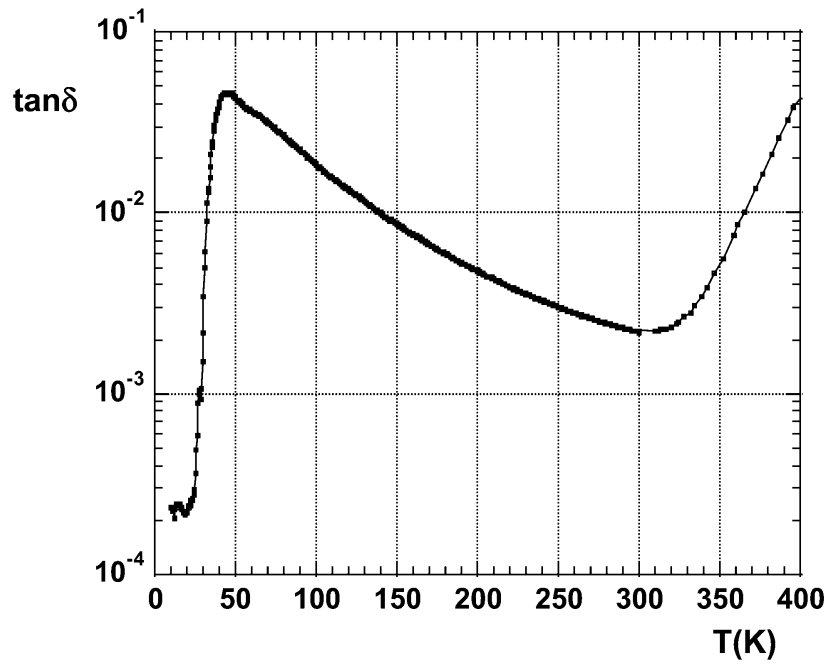

Fig. 8. Effective dielectric loss tangent versus temperature for silicon sample $\# 3$ at a frequency of approximately $4.98 \mathrm{GHz}$ employing $\mathrm{TE}_{011}$ mode in composite dielectric resonator.

frequency of the $\mathrm{TE}_{011}$ mode for the composite resonator with a PTFE sample is essentially the same as that without it. At cryogenic temperatures, PTFE losses are very low so that one can treat the $Q$-factor values of the resonator with a PTFE sample as being the same as the $Q$-factor values for an empty dielectric resonator. Additionally, the $Q$ factor of the empty cavity without the BZT resonator and quartz support was measured in order to evaluate the surface resistance changes of the silverplated cavity versus temperature. Results of $Q$-factor measurements and loss tangent determination for sample \#3 are shown in Figs. 7 and 8. It is seen that by employing a composite dielectric resonator, measurements are possible in the whole temperature range using the quasi- $\mathrm{TE}_{011}$ mode. The upper limit on measurement temperature for the composite resonator is determined by the resonator construction rather than by the dielectric losses in

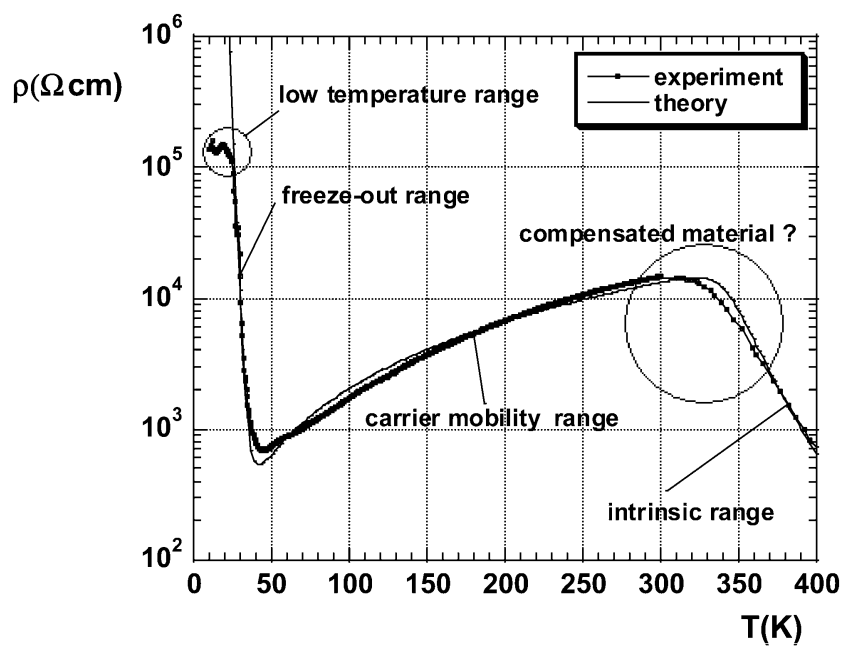

Fig. 9. Resistivity versus temperature extracted from measurements shown in Fig. 8 for silicon sample \#3 at a frequency of approximately $4.98 \mathrm{GHz}$. Parameters of silicon assumed in computations of theoretical curve are shown in Table II.

TABLE II

PARAMETERS OF SILICON ASSUMED IN THEORETICAL COMPUTATIONS OF RESISTIVITY

\begin{tabular}{ll}
\hline \hline $\begin{array}{l}\text { Acceptor ionization energy } \mathrm{E}_{\mathrm{a}}(\mathrm{eV}) \\
\text { (Boron) }\end{array}$ & 0.045 \\
\hline Acceptor concentration $\mathrm{N}_{\mathrm{a}}\left(\mathrm{cm}^{-3}\right)$ & $1.05 \times 10^{12}$ \\
\hline Electron mobility $\mathrm{v}_{\mathrm{e}}\left(\mathrm{cm}^{2} /(\mathrm{Vs})\right)$ & $1430 \times(\mathrm{T} / 300)^{-2.3}$ \\
\hline Hole mobility $\mathrm{v}_{\mathrm{h}}\left(\mathrm{cm}^{2} /(\mathrm{Vs})\right)$ & $470 \times(\mathrm{T} / 300)^{-1.76}$ \\
\hline Energy gap $\mathrm{E}_{\mathrm{g}}(\mathrm{eV})$ & $1.1785-9.025 \times 10^{-5} \times \mathrm{T}-3.05 \times 10^{-7} \times \mathrm{T}^{2}$ \\
\hline $\begin{array}{l}\mathrm{n}_{\mathrm{i}}-\text { intrinsic carrier concentration } \\
\text { at } 300 \mathrm{~K}\left(\mathrm{~cm}-{ }^{3}\right)\end{array}$ & $1.75 \times 10^{10}$ \\
\hline
\end{tabular}

silicon. For this structure, it is limited by the coaxial cables and the use of a tin solder.

Dielectric loss tangent values for samples \#1 and \#3 that are shown in Figs. 4 and 8 are similar when they are scaled with frequency. Measurements of dielectric loss tangents larger than $2 \times 10^{-3}$ are more accurate, employing the composite dieletric-resonator technique, while measurements of loss tangents smaller than $1 \times 10^{-3}$ are more accurate when the silicon sample stands alone as a dielectric resonator. In Fig. 9, resistivity values extracted from measurements of the loss tangents shown in Fig. 8 are presented. For comparison, we have evaluated theoretical resistivity values from well-known silicon resistivity models (e.g., see [15]). The best fit to experimental data has been obtained assuming the parameters of silicon given in Table II. It can be noticed that temperature dependence of resistivity, or dielectric loss tangent, can be satisfactory explained for temperatures larger than $25 \mathrm{~K}$ on the basis of well-understood theories of semiconductors. At temperatures above $25 \mathrm{~K}$, thermal energy is sufficient for partial ionization of Boron dopands, which become fully ionized at temperatures close to $45 \mathrm{~K}$. At temperatures from 45 to $300 \mathrm{~K}$, the number of free carriers is approximately constant and conductivity decreases predominantly due to decreasing hole mobility (in p-type semiconductor). If the temperature increases to approximately $300 \mathrm{~K}$, the number of additional free carriers generated through the forbidden energy gap becomes comparable to the number 
of holes due to ionized acceptors, and at temperatures above $350 \mathrm{~K}$, high-resistivity silicon becomes essentially intrinsic with an approximately equal number of holes and electrons.

The origin of dielectric losses at temperatures below $25 \mathrm{~K}$ is not yet clear and requires further investigations.

\section{CONCLUSIONS}

The dielectric losses of float-zone high-resistivity silicon have been measured between 10-400 $\mathrm{K}$ using two dielectric resonator measurement techniques. Using a novel composite dielectric-resonator configuration, measurements are possible even for samples with very low $Q$ factors. The composite dielectric-resonator technique can be easily adopted for measurements of arbitrary semiconductors, even those having much smaller resistivity than the high-resistivity silicon used here, by the appropriate choice of dielectric-resonator dimensions. Measurements at temperatures as high as $600 \mathrm{~K}$ will be possible by using appropriate coaxial cables with welded connectors and silica insulation.

\section{REFERENCES}

[1] J. G. Linhart, I. M. Templeton, and R. Dunsmuir, "A microwave resonant cavity method for measuring the resistivity of semiconducting materials," Brit. J. Appl. Phys., vol. 7, pp. 36-37, Jan. 1956.

[2] K. S. Champlin and R. R. Krongard, "The measurement of conductivity and permittivity of semiconductor spheres by an extension of the cavity perturbation method," IRE Trans. Microw. Theory Tech., vol. MTT-9, no. 11, pp. 545-551, Nov. 1961.

[3] K. S. Champlin, J. D. Holm, and G. H. Glover, "Electrodeless determination of semiconductor conductivity from TE $01^{\circ}$-Mode reflectivity," J. Appl. Phys., vol. 38, no. 1, pp. 96-98, Jan. 1967.

[4] K. S. Champlin and G. H. Glover, "Influence of waveguide contact on measured complex permittivity of semiconductors," J. Appl. Phys., vol. 37, no. 6, pp. 2355-2360, May 1966.

[5] S. Dmowski, J. Krupka, and A. Milewski, "Contactless measurement of silicon resistivity in cylindrical TE $\mathrm{TE}_{01 n}$ mode cavities," IEEE Trans. Instrum. Meas., vol. IM-29, no. 1, pp. 67-70, Mar. 1980.

[6] M. N. Afsar and H. Chi, "Millimeter wave complex refractive index, complex dielectric permittivity and loss tangent of extra high purity and compensated silicon," Int. J. Infrared Millim. Waves, vol. 15, no. 7, pp. 1181-1188, Jul. 1994.

[7] V. V. Parshin, R. Heidinger, B. A. Andreev, A. V. Gusev, and V. B. Shmagin, "Silicon as an advanced window material for high power gyrotrons," Int. J. Infrared Millim. Waves, vol. 16, no. 5, pp. 863-877, May 1995.

[8] H. Sobol, "Applications of integrated circuit technology to microwave frequencies," Proc. IEEE, vol. 59, no. 8, pp. 1200-1211, Aug. 1971.

[9] A. C. Reyes, S. M. El-Ghazaly, S. J. Dorn, M. Dydyk, D. K. Schroder, and H. Patterson, "Coplanar waveguides and microwave inductors on silicon substrates," IEEE Trans. Microw. Theory Tech., vol. 43, no. 9, pp. 2016-2021, Sep. 1995.

[10] R. A. Pucel, "Design considerations for monolithic microwave circuits," IEEE Trans. Microw. Theory Tech., vol. MTT-29, no. 6, pp. 513-534, Jun. 1981.

[11] J. Krupka, K. Derzakowski, B. Riddle, and J. Baker-Jarvis, "A dielectric resonator for measurements of complex permittivity of low loss dielectric materials as a function of temperature," Meas. Sci. Technol., vol. 9, pp. 1751-1756, Oct. 1998.

[12] J. Krupka, K. Derzakowski, A. Abramowicz, M. E. Tobar, and R. G. Geyer, "Whispering gallery modes for complex permittivity measurements of ultra-low loss dielectric materials," IEEE Trans. Microw. Theory Tech, vol. 47, no. 6, pp. 752-759, Jun. 1999.

[13] J. Krupka, K. Derzakowski, M. E. Tobar, J. Hartnett, and R. G. Geyer, "Complex permittivity of some ultralow loss dielectric crystals at cryogenic temperatures," Meas. Sci. Technol., vol. 10, pp. 387-392, Oct. 1999.
[14] J. Krupka, W.-T. Huang, and M.-J. Tung, "Complex permittivity measurements of low loss microwave ceramics employing higher order quasi-TE $\mathrm{TE}_{0 n p}$ modes excited in a cylindrical dielectric sample," Meas. Sci. Technol., vol. 16, no. 4, pp. 1014-1020, Apr. 2005.

[15] B. I. Shklovskii and A. L. Efros, Electronic Properties of Doped Semiconductors. Berlin, Germany: Springer, 1984, ch. 4.

[16] K. Y. Tsao and C. T. Sah, "Temperature dependence of resistivity and hole conductivity mobility in p-type silicon," Solid State Electron., vol. 19, no. 11, pp. 949-953, Nov. 1976.

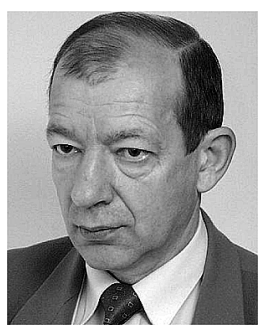

Jerzy Krupka (M'00-SM'01) was born in Cracow, Poland, in 1949. He received the M.Sc. (Hons.), Ph.D., and Habilitation degrees from the Warsaw University of Technology, Warsaw, Poland, in 1973, 1977 , and 1989, respectively.

Since 1973 he has been with the Institute of Microelectronics and Optoelectronics, Warsaw University of Technology, where he is currently a Professor. His research deals mainly with measurements of the electric and magnetic properties of materials at microwave. He was a leader and took part in several research projects on these subjects in the U.S., U. K., Australia, Germany, and France.

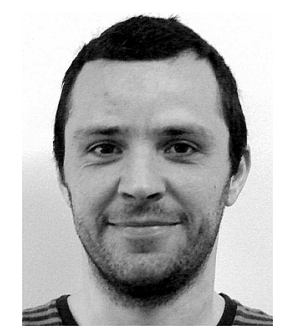

Jonathan Breeze received the B.Sc.(Hons.) degree in astrophysics from The University of Leeds, Leeds, U.K., in 1991.

He then joined the National Physical Laboratory, where he developed cryogenic sapphire dielectric resonators. In 1993, he joined Matria Marconi Space, as a Senior Design Engineer involved with passive microwave devices for spaceborne applications. While there, he also conducted research into high-temperature superconductor (HTS) and dieletric-resonator filters. Since 1998, he has been with the Centre for Physical Electronics and Materials, London South Bank University, London, U.K., where he is currently a Senior Research Fellow. His interests are microwave resonators and filters and the ab-initio quantum calculation of electrical properties of materials.

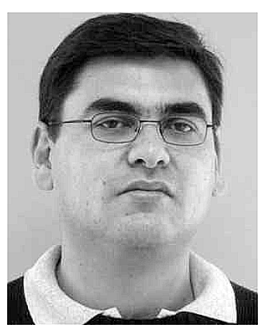

Anthony Centeno received the B.Eng. and Ph.D. degrees from the University of Wales, Wales, U.K., in in 1988 and 1994, respectively.

From 1992 to 1994, he was a Research Assistant with the University of Bradford. From 1994 to 1996, he was a Senior Microwave Engineer with Matra Marconi Space Ltd., Portsmouth, U.K. From 1996 to 2000, he was a Senior Scientist with the Defence Evaluation and Research Agency. From 2000 to 2003, he was an Assistant Professor with the Universiti Nottingham Malaysia. He has also been a Visiting Academic with the Universiti Technologi Malaysia and the Malaysian Military Academy. He is currently a Senior Lecturer with London South Bank University, London, U.K. His research interests are in the area of applied electromagnetics.

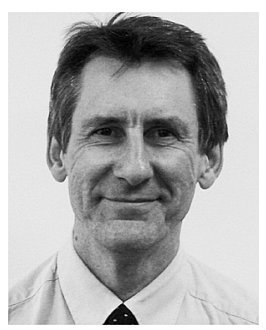

Neil Alford received the B.Sc. (Hons.) degree in geology from St. Andrews University, St. Andrews, U.K., in 1973, and the Ph.D. degree in materials science from Queen Mary College, London, U.K., in 1979.

From 1979 to 1981, he was a Post-Doctoral Researcher with Oxford University. In 1981, he joined ICI, where he was involved with projects concerning the processing and fracture of brittle materials, layered composites, and the mechanical and electrical properties of perovskite ceramics, specifically HTSs. Since 1994, he has been a Professor of physical electronics and materials with London South Bank University, London, U.K., where he is currently involved with the development of HTS magnetic resonance imaging (MRI) receive coils, microwave dielectrics, novel signal transformers, and ferroelectric thin films. 
Thomas Clausen received the M.Sc. and Ph.D. degrees from the Technical University of Denmark, Lyngby, Denmark, in 1990 and 1993, respectively. His doctoral thesis concerned metal-semiconductor interfaces.

He was then a Post-Doctoral Researcher and Associate Professor in various research fields related to semiconductors, superconductors, and semiconductor-superconductor interfaces. Since 1998, he has been with different companies in a variety of positions, the most recent being Product Manager with Topsil Semiconductor Material A/S, Frederikssund, Denmark. Since December 2005, he has been with PBI-Dansensor, Ringstead, Denmark, as Project Manager for sensor research and development. He has authored or coauthored over 20 papers in refereed journals.
Leif Jensen received the B.Sc. degree from Århus Teknikum, Århus, Denmark, in 1984.

He has worked as an Electronic Engineer. Since 1986, he has been involved with float-zone techniques and silicon crystal growth with Topsil Semiconductor Material A/S, Frederikssund, Denmark, where since 1992, he has been a Research and Development Manager. 